\title{
A Reply to Mueller (2018) Supply Chain Collaboration: Further Insights into Incentive Alignment in the Beer Game Scenario
}

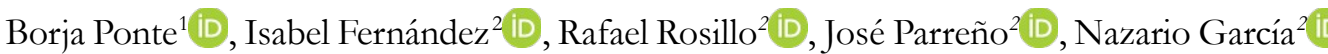 \\ ${ }^{1}$ The Open University (United Kingdom), ${ }^{2}$ University of Oviedo (Spain) \\ borja.ponte-blanco@open.ac.uk,.ifq@uniovi.es,rosillo@uniovi.es,parreno@uniovi.es,ngarcia@uniovi.es
}

Received: January 2018

Accepted: June 2018

\begin{abstract}
:
Purpose: We expand a previous discussion in this journal by proposing a new solution concept, based on game theory, for profit allocation with the aim of aligning incentives in collaborative supply chains.

Design/methodology/approach: Through the Gately's notion of propensity to disrupt, we minimize the desire of the nodes to leave the grand coalition in the search of a self-enforcing allocation mechanism.

Findings: We discuss the benefits and limitations of this solution in comparison with more established alternatives (e.g. nucleolus and Shapley value). We show that it considers the bargaining power of the nodes, but it may not belong to the core.
\end{abstract}

Originality/value: Finding a fair and self-enforcing scheme for incentive alignment, and specifically profit allocation, is essential to ensure the long-term sustainability of collaborative supply chains.

Keywords: beer game, game theory, Gately point, incentive alignment, profit allocation, supply chain collaboration

\section{Introduction}

In 2016, we (Ponte, Fernández, Rosillo, Parreño \& García, 2016) investigated in this journal how game-theoretical concepts and ideas suggest an appropriate framework to align incentives in supply chains through the design of a fair and self-enforcing mechanism for allocating the net profit derived from collaborative solutions. From this perspective, we defined three conditions (efficiency, individual rationality, and coalition rationality) to be verified in viable profit allocations, and proposed a mechanism for profit allocation based on the notion of the nucleolus (Schmeidler, 1969). This mechanism allocates the profit among the different supply chain partners by maximizing the satisfaction of the unhappiest coalition. We think that this solution concept fits very well with the essence of the incentive alignment problem in collaborative supply chains (see e.g. Simatupang \& Sridharan, 2005). To illustrate our proposal, we developed a numerical example based on the widely-used Beer Game scenario (Goodwin \& Franklin, 1994). We showed that it clearly outperforms traditional methods for profit allocation, which despite their simplicity are often used in practice (see e.g. Liu, Wu \& Xu, 2010), in terms of compensation fairness.

Recently, Mueller (2018) extended the discussion initiated by our previous paper from different perspectives. First, they presented and discussed additional game-theoretic properties that can be useful for ensuring the robustness of the profit allocation in collaborative supply chains. Second, they clarified the mathematical definition of the nucleolus through the concept of lexicographic order of a specific allocation. In this sense, the solution they suggested not only maximizes the satisfaction of the unhappiest coalition but, when several possible allocations verify this aim, it also looks at (maximizing the satisfaction of) the second unhappiest coalition, the third one, and so on. It should be noted that the computation of this solution would require solving a sequence of nested 
optimization problems. Finally, they presented a new solution concept for this problem, i.e. the Lorenz set (see e.g. Hougaard, Peleg \& Thorlund-Petersen, 2001), based on the concept of Lorenz dominance (Bishop, Formby \& Smith, 1991), which looks at the concentration of wealth in the coalition. Mueller (2018) employed the same numerical example to illustrate their proposal and discussed the different solutions.

We truly appreciate the authors' efforts in continuing the avenue that we opened in our prior work. Through the previously highlighted lines of contribution, we believe that they provide relevant insights into the analysis of the value of game theory concepts in the field of supply chain collaboration. In this new article we would like to continue the discussion by proposing a new solution concept for the problem of profit allocation in collaborative supply chains. This is based on the Gately point (Gately, 1974; Straffin, 1993), which aims to minimize the maximum propensity to deviate from the collaborative behavior in the supply chain. We present this new mechanism for incentive alignment, discuss why it fits with the essence of the profit allocation problem, and illustrate its application to the same numerical example in the Beer Game scenario. Through the comparison with the previous benchmark alternatives, we aim to gain insight into the usefulness and limitations of the different solution concepts.

\section{Characterization of the Gately solution}

Gately (1974) introduced the concept of propensity to disrupt a coalition to explore the distribution of the gains among three partners in a problem concerning regional cooperation in planning investment in electric power. Shortly after, this concept was extended to $n$-person games, see e.g. Littlechild and Vaidya (1976).

Consider a grand coalition of $\mathrm{n}$ partners $N=\{1,2, \ldots, n\}$, where the value function $v(N)$ expresses the joint gains obtained by the coalition and $v(C)$, where $C \subseteq N$, represents the joint gains obtained by a specific coalition $C$ formed by a subset of the partners. For a given payoff vector $x=\left(x_{1}, x_{2}, \ldots, x_{n}\right)$, the partner i's propensity to disrupt the grand coalition $p d_{i}(x)$ is defined as the ratio of how much the other nodes would lose if $i$ abandons the coalition (the numerator) to how much this partner would lose if breaking away (the denominator). This idea is expressed by Equation (1), see e.g. Straffin (1993).

$$
\operatorname{pd}_{i}(x)=\frac{\left\{\sum_{k \in\{N-i\}} x_{k}\right\}-v(N-i)}{x_{i}-v(i)}
$$

We believe that this concept fits very well with the essence of the incentive alignment problem within supply chain collaboration. Note that a denominator lower than 0 reveals that the supply chain node $i$ would increase its net profit by abandoning the grand coalition and operating alone, while a numerator lower than 0 means that the rest of the nodes would increase their joint net profit if the node $i$ leaves the coalition. Any of both alternatives would imply that the payoff vector $\mathrm{x}$ is not a viable coalition, as this allocation of the net profit would not satisfy all the partners. In addition, and in line with Shi, Wang, Zhang, Zhang and Zhang (2016), positive propensities to disrupt may be interestingly interpreted as indicative of the bargaining power of the different supply chain nodes for a specific payoff vector $x$, and hence provides an idea of the strength of the motivation of the different nodes for abandoning the coalition $N$. The higher $p d_{i}(x)$, the less comfortable the node $i$ will feel in the grand coalition. Note that $p d_{i}(x)>>1$ would indicate that the participation of this node in the coalition generates much more net profit for the rest of the nodes than for itself.

Underlying this concept, an interesting idea emerges as a solution mechanism for cooperative games: selecting the payoff vector that minimizes the maximum propensity to disrupt of the different nodes. This is known as the Gately point; see e.g. Dhamal, Vallam and Narahari (2017) and Tatarczak (2017) for recent studies using this concept. Straffin (1993) highlights that an observation that holds in general for this solution concept is that "the way to minimize the largest propensity to disrupt is to make all of the propensities to disrupt equal". This leads to a

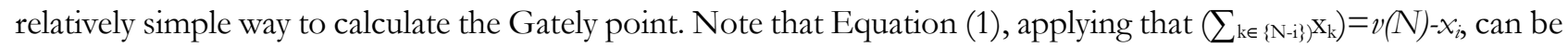
transformed into Equation (2).

$$
p d_{i}(x)=\frac{v(N)-x_{i}-v(N-i)}{x_{i}-v(i)}=\frac{v(n)-(v(N-i)+v(i))}{x_{i}-v(i)}-1
$$


Note that by trying to equalize the propensity to disrupt of the different nodes, which quantifies the desire of the nodes to leave the grand coalition, the Gately solution not only looks at compensation fairness but also at selfenforcement, which fits perfectly with the notion of incentive alignment in collaborative supply chains defended by Simatupang and Sridharan (2005).

From Equation (2), all the $p d_{i}(x)$, can be made equal by selecting $x_{i}-v(i)$ in proportion to $v(N)-(v(N-i)+v(i))$. Note that assuming the superadditivity property holds in a supply chain context (see Ponte et al., 2016), $v(N)-(v(N-i)+v(i)) \geq 0$, which quantifies the net profit increase due to the participation of node $\mathrm{i}$ in the collaborative solution. This leads to Equation (3) for the Gately point.

$$
x_{i}^{G}=v(i)+\frac{v(N)-(v(N-i)+v(i))}{\sum_{k \in N}[v(N)-(v(N-k)+v(k))]} \cdot\left(v(N)-\sum_{j \in N} v(j)\right)
$$

This implies that, in the Gately solution, each supply chain node captures a portion of the surplus generated by collaboration (in comparison with the different nodes acting alone) proportional to the contribution of its participation to increase the overall net profit of the supply chain.

\section{A numerical example based on the Beer Game scenario}

In this article, we use the numerical example based on supply chain collaboration in the Beer Game scenario developed by Ponte et al. (2016) and also used by Mueller (2018). The characteristic function of this profit allocation problem is provided in Table 1. We refer to the former article for more details on the net profit of the different coalitions in this numerical example.

\begin{tabular}{|c|c|c|c|}
\hline no collaboration & 2-node coalitions & 3-node coalitions & 4-node coalitions \\
\hline $\mathrm{v}(1)=100$ & $\mathrm{v}(12)=400$ & $\mathrm{v}(123)=800$ & $\mathrm{v}(1234)=1,500$ \\
\hline $\mathrm{v}(2)=200$ & $\mathrm{v}(13)=450$ & $\mathrm{v}(124)=850$ & \\
\hline $\mathrm{v}(3)=300$ & $\mathrm{v}(14)=600$ & $\mathrm{v}(134)=1,250$ & \\
\hline $\mathrm{v}(4)=400$ & $\mathrm{v}(23)=550$ & $\mathrm{v}(234)=1,050$ & \\
\hline & $\mathrm{v}(24)=650$ & & \\
\hline & $\mathrm{v}(34)=750$ & & \\
\hline
\end{tabular}

Table 1. Net profit for the different possible coalitions in the supply chain

In this section, we first obtain and discuss the Gately solution for this case study. Second, we compare this solution against the benchmark alternatives proposed by Ponte et al. (2016) and Mueller (2018). From this perspective, we analyze the differences between the different mechanisms for profit allocation in collaborative supply chains, looking at their usefulness and limitations.

\subsection{Gately solution for profit allocation}

Applying Equation (3) leads to the following Gately solution for the problem we have posed. Note that we detail the different terms to facilitate the conceptual understanding of the profit allocation.

$$
\begin{array}{ll}
x_{1}^{G}=100+\frac{1,500-(1,050+100)}{350+50+350+300}(1,500-1,000)=266.67 & x_{3}^{G}=300+\frac{1,500-(850+300)}{350+50+350+300}(1,500-1,000)=466.67 \\
x_{2}^{G}=200+\frac{1,500-(1,250+200)}{350+50+350+300}(1,500-1,000)=223.81 & x_{4}^{G}=400+\frac{1,500-(800+400)}{350+50+350+300}(1,500-1,000)=542.86
\end{array}
$$

Applying Equation (1), it can be easily checked that this profit allocation $x^{G}=(266.67,223.81,466.67,542.86)$ generates the same propensity to disrupt for the four supply chain nodes. 


$$
\begin{aligned}
& p d_{1}\left(x^{G}\right)=\frac{(223.81+466.67+542.86)-1,050}{266.67-100}=1.1 \\
& p d_{2}\left(x^{G}\right)=\frac{(266.67+466.67+542.86)-1,250}{223.81-200}=1.1
\end{aligned}
$$$$
p d_{3}\left(x^{G}\right)=\frac{(266.67+223.81+542.86)-850}{466.67-300}=1.1
$$$$
p d_{4}\left(x^{G}\right)=\frac{(266.67+223.81+466.67)-800}{542.86-400}=1.1
$$

\subsection{Comparison of the various methods for profit allocation}

Ponte et al. (2016) compared the solution they proposed, which allocates the profit to maximize the satisfaction of the unhappiest coalition, with two benchmark alternatives: the equal and the proportional distributions of the surplus. They showed that, while these benchmark solutions generate profit allocations that may leave some internal coalitions unsatisfied (and hence are not sustainable in long term), their proposed solution satisfies all the possible allocations. Mueller (2018) showed that this stable and fair solution can be improved by looking at the lexicographic order of the satisfaction vector according to the mathematical definition of the nucleolus. They also presented traditional game-theoretic solutions based on the Shapley value (Shapley, 1953) and the Lorenz set (Hougaard et al., 2001). The former allocates the net profit according to the marginal contribution of each node; however, they showed that this solution does not belong to the core in this case study, and hence may not be practically viable. The latter is interesting from the perspective that it minimizes the inequality of the profit allocation in the collaborative solution.

Following this line of argument, we now evaluate the Gately solution against the nucleolus, Shapley and Lorenz solutions. The net profit allocations according to these three game-theoretic mechanisms are synthetized in Table 2.

In order to compare the four solution mechanisms, we first use the game-theoretic notion of excess (Schmeidler, 1969). This can be used to quantify the satisfaction of the different possible coalitions in the supply chain; note that satisfaction $=-$ excess. In this sense, the satisfaction can be expressed as the difference between how much the nodes of the coalition $C$ are earning in the grand coalition $N$ and how much they would be earning by acting alone in the coalition $C$. The satisfaction of the various coalitions in the three solution mechanisms can be seen in Table 3 . In every case, we highlight (in bold) the result of the most unsatisfied coalition.

The Shapley solution may be a fair mechanism from the perspective that it looks at the contribution of each node to the overall profit. Despite this, its implementation in practice may not be viable as a coalition of nodes 1,3 , and 4 would generate more money that what these nodes obtain in the larger coalition. This means that, as pointed out by Mueller (2018), this solution does not belong to the core. On the other hand, the Lorenz solution selects the allocation within the core that minimizes the inequality. However, this is achieved by making equal to 0 the satisfaction of the unhappiest coalition. It implies that node 2 is capturing all the increase that this node is contributing to the collaboration, which may not be acceptable for its supply chain partners (why would they be interested in node 2 joining the grand coalition if this does not have a positive impact on them?). On the contrary, both the Gately and the nucleolus solutions generate a positive satisfaction for all the possible coalitions in this specific case study. For this reason, both solution concepts can be interpreted as viable in this case study. As can be expected (given that this is the goal of the nucleolus allocation), the nucleolus allocation makes the unhappiest coalition more satisfied. Nonetheless, note that the difference is relatively small (23.81 against 25).

\begin{tabular}{|c|c|c|c|c|}
\hline $\begin{array}{c}\text { Supply chain } \\
\text { node }\end{array}$ & Gately solution & $\begin{array}{c}\text { Nucleolus solution } \\
\text { (Mueller, 2018) }\end{array}$ & $\begin{array}{c}\text { Shapley solution } \\
\text { (Mueller, 2018) }\end{array}$ & $\begin{array}{c}\text { Lorenz solution } \\
\text { (Mueller, 2018) }\end{array}$ \\
\hline Factory (1) & 266.67 & 291.67 & 262.50 & 416.67 \\
\hline Distributor (2) & 223.81 & 225 & 254.17 & 250 \\
\hline Wholesaler (3) & 466.67 & 441 & 445.83 & 416.67 \\
\hline Retailer (4) & 542.86 & 541 & 537.50 & 416.67 \\
\hline Total & 1,500 & 1,500 & 1,500 & 1,500 \\
\hline
\end{tabular}

Table 2. Profit allocation according to the various game-theoretic mechanisms 


\begin{tabular}{|c|c|c|c|c|}
\hline Coalition & Gately solution & Nucleolus solution & Shapley solution & Lorenz solution \\
\hline 1 & 166.67 & 191 & 162.5 & 316.67 \\
\hline 2 & $\mathbf{2 3 . 8 1}$ & $\mathbf{2 5}$ & 54.17 & 116.67 \\
\hline 3 & 166.67 & 141.67 & 145.83 & 16.67 \\
\hline 4 & 142.85 & 141.67 & 137.5 & 266.67 \\
\hline $1-2$ & 90.48 & 116.67 & 116.67 & 383.33 \\
\hline $1-3$ & 283.33 & 283.33 & 258.33 & 233.33 \\
\hline $1-4$ & 209.52 & 233.33 & 150 & 116.67 \\
\hline $2-3$ & 140.48 & 116.67 & 141.67 & 83.33 \\
\hline $2-4$ & 116.67 & 116.67 & 233.33 & 283.33 \\
\hline $3-4$ & 259.52 & 233.33 & 162.5 & 233.3 \\
\hline $1-2-3$ & 157.14 & 158.33 & 204.17 & $\mathbf{0}$ \\
\hline $1-2-4$ & 183.33 & 208.33 & 187.5 & 33.33 \\
\hline $1-3-4$ & 26.19 & $\mathbf{2 5}$ & 158.33 & \\
\hline $2-3-4$ & 183.33 & & & \\
\hline
\end{tabular}

Table 3. Satisfaction of the coalitions various game-theoretic mechanisms

\begin{tabular}{|c|c|c|c|c|}
\hline Supply chain node & Gately solution & Nucleolus solution & Shapley solution & Lorenz solution \\
\hline Factory (1) & 1.1 & 0.83 & 1.15 & 0.11 \\
\hline Distributor (2) & 1.1 & 1 & -0.08 & 0 \\
\hline Wholesaler (3) & 1.1 & 1.47 & 1.4 & 2 \\
\hline Retailer (4) & 1.1 & 1.12 & 1.18 & 17 \\
\hline
\end{tabular}

Table 4. Propensity to disrupt of the coalitions various game-theoretic mechanisms

We can also compare the three solutions based on the concept of propensity to disrupt that we have previously defined. Table 4 present these results.

The problems of the Shapley solution in this case study become evident through a propensity to disrupt (of the distributor) lower than 0 . As we discussed before, this occurs as the participation of this node in the grand coalition decreases the joint net profit of the other nodes. Meanwhile, the propensity to disrupt of this node under the Lorenz solution is 0 , which reveals that its participation is generating no benefit in its supply chain partners. In line with the previous analysis, both the Gately and the nucleolus concepts offer viable solutions in this case study. The main difference is that while the Gately point generates the same propensity to disrupt for all the nodes, the nucleolus generates different propensities. Note that in this case, some of the propensities to disrupt are lower than 1 and other are higher than 1. It implies that while the participation of the wholesaler (e.g.) in the grand coalition generates a higher net profit to the factory than to itself, the participation of the factory generates a higher net profit to itself than to the other nodes. From the perspective that it may be problematic in some practical contexts, the Gately point suggests minimizing the maximum propensity to disrupt, which makes all them equal.

Finally we measure the Euclidean length of the different allocations by Equation (4).

$$
\|x\|=\sqrt{\sum_{k \in N} x_{k}^{2}}
$$

As discussed by Mueller (2018), the indicator provides information on the concentration of wealth in the supply chain. The lower this indicator, the less inequality. Table 5 presents these results. By its own definition, the Lorenz solution minimizes the Euclidean length for those imputations within the core. Note that the Shapley solution, 
although it does not belong to the core, offers a higher length. Both the Gately and the nucleolus solutions provide a similar performance in terms of this criterion, although it should be mentioned that the nucleolus provides a better performance according to this metric. It may be interpreted in the following manner: considering the bargaining power of the different nodes, according to the Gately solution, slightly increases the inequality in the supply chain.

\begin{tabular}{|c|c|c|c|}
\hline Gately solution & Nucleolus solution & Shapley solution & Lorenz solution \\
\hline 796.04 & 790.04 & 788.15 & 763.62 \\
\hline
\end{tabular}

Table 5. Euclidean length of the allocations of the game-theoretic mechanisms

\section{Concluding notes}

This research aims to contribute to the discussion initiated by Ponte et al. (2016) and enriched by Mueller (2018) on the value of game-theoretic ideas for aligning incentives in collaborative supply chains. From this perspective, we introduce a new solution concept based on Gately's notion of propensity to disrupt. Through a numerical case study built on the four-node Beer Game scenario, we compare this solution concept against more established mechanisms, like the Shapley value and the nucleolus. We discuss the strengths and weaknesses of each alternative.

The main interest of the solution we propose in this article is that it minimizes the desire of the supply chain nodes to leave the overall collaboration. This is especially interesting in a supply chain context, as it somewhat considers the bargaining power of the different echelons. Therefore, it accounts for both the fairness and the self-enforcing properties of the allocation of the gains obtained from the collaboration. Another interesting advantage of this alternative is that its computation is relatively simple in comparison with other alternatives, which may make it more applicable in practice in a dynamic manner.

Nonetheless, it is pertinent to underline a relevant drawback of this alternative. While the nucleolus always belongs to the core (as long as it is non-empty), the Gately solution may not belong to it in some scenarios (Straffin, 1993). It did not happen in our case study, but it may occur in some cases, especially when the core is small and/or the number of nodes is high. Nevertheless, the same idea can be extended to consider the propensities to disrupt of all possible coalitions in the supply chain (and not only those of the individual nodes) and minimizing the largest of these (in the same manner as in the nucleolus) to ensure that the solution belongs to the core. This solution has been labeled as the disruption nucleolus (see e.g. Massol \& Tchung-Ming, 2010). Exploring the advantages and limitations of this alternative in a supply chain context can be pointed out as an interesting avenue for future research.

\section{Declaration of Conflicting Interests}

The authors declared no potential conflicts of interest with respect to the research, authorship, and/or publication of this article.

\section{Funding}

The authors received no financial support for the research, authorship, and/or publication of this article.

\section{References}

Bishop, J. A., Formby, J. P., \& Smith, W. J. (1991). Lorenz dominance and welfare: Changes in the US distribution of income, 1967-1986. Review of Economics and Statistics, 134-139. https://doi.org/10.2307/2109695

Dhamal, S., Vallam, R. D., \& Narahari, Y. (2017). Modeling Spread of Preferences in Social Networks for Samplingbased Preference Aggregation. IEEE Transactions on Network. Science and Engineering, in press. https://doi.org/10.1109/TNSE.2017.2772878

Gately, D. (1974). Sharing the gains from regional cooperation: A game theoretic application to planning investment in electric power. International Economic Review, 195-208. https://doi.org/10.2307/2526099 
Goodwin, J. S., \& Franklin, S. G. (1994). The beer distribution game: using simulation to teach systems thinking. Journal of Management Development, 13(8), 7-15. https://doi.org/10.1108/02621719410071937

Hougaard, J.L., Peleg, B., \& Thorlund-Petersen, L. (2001). On the set of Lorenz-maximal imputations in the core of a balanced game. International Journal of Game Theory, 30(2), 147-165. https://doi.org/10.1007/s001820100070

Littlechild, S. C., \& Vaidya, K. G. (1976). The propensity to disrupt and the disruption nucleolus of a characteristic function game. International Journal of Game Theory, 5(2-3), 151-161. https://doi.org/10.1007/BF01753316

Liu, P., Wu, Y., \& Xu, N. (2010). Allocating collaborative profit in less-than-truckload carrier alliance. Journal of Service Science and Management, 3(1), 143-149. https://doi.org/10.4236/jssm.2010.31018

Massol, O., \& Tchung-Ming, S. (2010). Cooperation among liquefied natural gas suppliers: Is rationalization the sole objective?. Energy Economics, 32(4), 933-947. https://doi.org/10.1016/j.eneco.2010.02.008

Mueller, D. (2018). A reply to Ponte et al. (2016) - Supply chain collaboration: Some comments on the nucleolus of the Beer Game. Journal of Industrial Engineering and Management, 11(3), 528-534.

https://doi.org/10.3926/jiem.2430

Ponte, B., Fernández, I., Rosillo, R., Parreño, J., \& García, N. (2016). Supply chain collaboration: a game-theoretic approach to profit allocation. Journal of Industrial Engineering and Management, 9(5), 1020-1034.

https://doi.org/10.3926/jiem.2084

Schmeidler, D. (1969). The nucleolus of a characteristic function game. Journal of Applied Mathematics, 17(6), 1163-1170. https://doi.org/10.1137/0117107

Shapley, L.S. (1953). A value for n-person games. In Kuhn, H.W., \& Tucker, A.W. (Eds.), Contributions to the theory of games (II) (307-317). Princeton: Princeton Univ. Press. https://doi.org/10.1515/9781400881970-018

Shi, G. M., Wang, J. N., Zhang, B., Zhang, Z., \& Zhang, Y. L. (2016). Pollution control costs of a transboundary river basin: Empirical tests of the fairness and stability of cost allocation mechanisms using game theory. Journal of Environmental Management, 177, 145-152. https://doi.org/10.1016/j.jenvman.2016.04.015

Simatupang, T. M., \& Sridharan, R. (2005). An integrative framework for supply chain collaboration. International Journal of Logistics Management, 16(2), 257-274. https://doi.org/10.1108/09574090510634548

Straffin, P. D. (1993). Game theory and strategy (Vol. 36). MAA.

Tatarczak (2017). Allocative Collaborative Profit in Supply Chains. In Yu. G. Evtushenko, M. Yu. Khachay, O. V. Khamisov, Yu. A. Kochetov, V.U. Malkova, M.A. Posypkin (Eds.): Proceedings of the OPTIMA-2017 Conference, Petrovac, Montenegro.

\section{Journal of Industrial Engineering and Management, 2018 (www.jiem.org)}

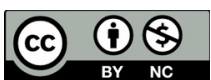

Article's contents are provided on an Attribution-Non Commercial 4.0 Creative commons International License. Readers are allowed to copy, distribute and communicate article's contents, provided the author's and Journal of Industrial Engineering and Management's names are included. It must not be used for commercial purposes. To see the complete license contents, please visit https://creativecommons.org/licenses/by-nc/4.0/. 